\title{
Neue Seuchen, alte Fragen
}

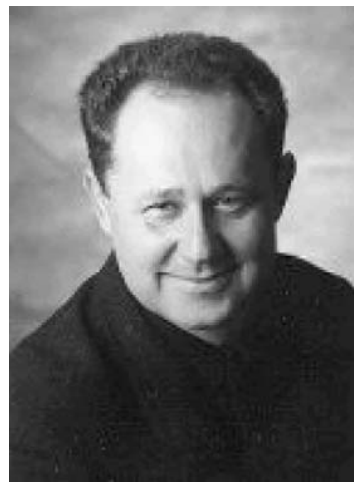

Prof. Dr. Manfred Wildner
Bibliografie

DOI http://dx.doi.org/ 10.1055/s-0031-1277149 Gesundheitswesen 2011; 73: 333-334

(c) Georg Thieme Verlag KG Stuttgart · New York ISSN 0941-3790

Korrespondenzadresse Prof. Dr. M. Wildner Bayerisches Landesamt für Gesundheit und Lebensmittelsicherheit Veterinärstraße 2 85762 Oberschleißheim Manfred.Wildner@Igl.bayern.de
Weltweit werden jährlich Typhus-Neuerkrankungen in zweistelliger Millionenhöhe beobachtet, mit Hunderttausenden Toten: Eine Geißel vor allem in Ländern mit unzureichenden hygienischen Bedingungen. „In Deutschland konnte die Zahl der Erkrankungen seit 1951 durch eine erhebliche Verbesserung der hygienischen Bedingungen stark vermindert werden“, schreibt das renommierte Robert Koch-Institut. „Entsprechend der Meldepflicht nach IfSG wurden im Jahr 2006 an das RKI 75 Fälle von Typhus abdominalis übermittelt“ [1]. Eine Erfolgsgeschichte. Gleiches gilt zumindest in Deutschland für Lepra, Tuberkulose, Poliomyelitis und eine Vielzahl weiterer übertragbarer Krankheiten. Am 5. Juni 1981 wird eine Fallserie von 5 Patienten mit Pneumocystis carinii-Infektionen aus dem Cedars-Mount SinaiHospital in Los Angeles veröffentlicht - der erste wissenschaftliche Bericht zur erworbenen Immunschwächekrankheit AIDS (Acquired Immune Deficiency Syndrome) [2]. Innerhalb der seither vergangenen 30 Jahre haben sich über 60 Millionen Menschen mit dem Erreger, dem HI-Virus, infiziert, 25 Millionen Menschen starben. In Deutschland wurden dem RKI für das Jahr 2010 insgesamt knapp 3000 neu diagnostizierte HIVInfektionen gemeldet. In 6 von 7 Fällen waren Männer betroffen, überwiegend mit homosexueller Orientierung, eine von 10 Infektionen trat bei Personen aus Herkunftsländern mit hoher HIV-Prävalenz auf, intravenöser Drogengebrauch wird dem gegenüber bei einem von 20 Fällen als ursächlich angesehen [3]. Wobei die Frage nach Ursächlichkeiten alles andere als trivial ist. Eine Misserfolgsgeschichte?

Als Rudolf Virchow (1821-1902), der begabte junge Absolvent der preußischen (Militär)Akademie Pépinière im Revolutionsjahr 1848 den Auftrag bekam, die Fleckfieberepidemie in Oberschlesien zu untersuchen, war die epidemiologische Lage auch in Deutschland durchaus prekär: Schon seit 2 Jahren grassierte dort der „Hungertyphus“. Die Beschreibung der himmelschreienden sozialen Umstände und der dadurch bedingten sozialen Unruhen sind durch Gerhart Hauptmanns Drama „Die Weber“ (1893) Weltliteratur. Die Empfehlungen des damals 27 jährigen Privatdozenten Rudolf Virchow in der Aufarbeitung der oberschlesischen Typhusepidemie sorgten für nachhaltige Verstimmung bei seinen Auftraggebern: volle und uneingeschränkte Demokratie als Voraussetzung für Freiheit und Wohlstand für die etwa eineinhalb Millionen Armen, Selbstverwaltung, die Trennung von Staat und Kirche, die Verbesserung der Landwirtschaft, den Übergang der Steuerlasten von den Armen auf die Reichen, den Bau von Straßen und die Gründung von Genossenschaften [4].

Hoppla, Herr Virchow: war das nicht sehr weit hergeholt? Hat Sie Ihr revolutionärer Eifer des Jahres 1848 nicht etwas über das Ziel hinausschießen lassen? Empfehlungen zur Hygiene, zur Therapie, zu Maßnahmen der Infektionskontrolle in der Zuständigkeit einer Medizinischen Polizei wären doch wohl angebrachter gewesen als ein derartiger gesellschaftspolitischer Rundumschlag! So etwa müssen (auch) die im preußischen Staat zuständigen Amtsträger gedacht haben, die letztlich seine Entlassung aus dem Staatsdienst verfügten. Die Universität im bayerischen Würzburg war Nutznießerin dieser Entscheidung, welche den Genius gerne von 1849 bis 1856, dem Jahr seiner Rückkehr nach Berlin, bei sich aufnahm. Auch wenn Virchows Genie im allgemeinen mit der von ihm begründeten Zellularpathologie in Zusammenhang gebracht wird, sind doch auch seine Beiträge zur Sozialmedizin unvergessen: „Die Medizin ist eine soziale Wissenschaft, und die Politik ist weiter nichts, als Medizin im Großen“, lautet eine seiner bekanntesten Äußerungen, die in einem freiheitlichen Staat auch problematisch interpretiert werden kann [5]. Und so deuten für ihn die Seuchen auf „Mängel, welche durch die staatliche und gesellschaftliche Gestaltung erzeugt werden“ [6]. Sind demnach Seuchen gesellschaftliche Symptome, welche in eine bevölkerungsmedizinische Diagnostik Eingang finden sollten?

Dieses konsequent auf Systemzusammenhänge gerichtete analytisch-kausale Denken Rudolf Virchows, welches den Schritt von den proximalen biologischen Ursachen zu den mehr distal gelegenen sozialen und politischen Ursachen vollzieht, also in Ursachenketten, Verursachungsnetzen und „Verstrickungen“ denkt, ist noch immer zukunftsweisend. Parallelen zum modernen Ansatz des 1998 verstorbenen ehemaligen Direktors des WHO-Programms zur Bekämpfung von AIDS, Jonathan Mann, sind deutlich. „Es ist für einzelne Wissenschaftler und wissenschaftliche Institutionen immer sicherer, AIDS als eine virale übertragbare Krankheit zu konzipieren [...] Dr. Mann stellte dieses Thema dem gegenüber in den Kontext der Menschenrechte, strukturierte es als ein Thema globaler und universeller Rechte“ [7]. Diskriminierungen können Sprache und ethnischen Hintergrund betreffen, Geschlecht und sexuelle Orientierung, Alter und gesundheitlichen Zustand, berufliche und soziale Position - diese Aufzählung ist nicht abschließend. Dieser Ansatz, 
AIDS als Krankheit und die an AIDS Erkrankten aus der Diskriminierung zu holen und die Bürde dieser Krankheit als Herausforderung an die menschliche Gemeinschaft, wenn sie denn das Attribut der Menschlichkeit mit Würde tragen will, zu begreifen, wartet noch immer auf seine weitere Umsetzung. Sicher auch deswegen, weil das Denken in Ursachenketten spätestens nach dem dritten oder vierten „warum“ unbequeme Fragen an uns alle richtet. Wenn wir über i.v.-Drogen und homo- wie auch heterosexuelle Übertragungswege in der Prostitution nachdenken, sind größere gesellschaftliche Themen berührt. Was treibt Menschen in die Prostitution, was sind die Realitäten des Menschenhandels bis vor unsere Haustüren? Was sind ihre Hintergründe? Welche Belohnungs- oder Korruptionssysteme stützen sich auf die Prostitution und stützen damit die Prostitution? Was sind die Hintergründe des Drogenanbaus, wer verdient an seiner Organisation, seiner Transportlogistik? Was lässt Menschen empfänglich für die Verführungen des Drogenkonsums werden? Welche Rolle spielen Einkommens- und Bildungsarmut - bei uns und in den Drogenherkunftsländern? Welche Verantwortlichkeiten bestehen zwischen Völkern und Nationen? Welche der bevölkerungsmedizinisch „therapeutischen“ Empfehlungen Rudolf Virchows aus dem Jahr 1848 greifen heute noch immer: volle und uneingeschränkte Demokratie als Voraussetzung für Freiheit und Wohlstand, Selbstverwaltung, die Verbesserung der Landwirtschaft, der Übergang der Steuerlasten von den Armen auf die Reichen, der Bau von Straßen und die Gründung von Genossenschaften? In wieweit sind wir bereit, uns auf das Bild eines Netzwerkes allen Lebendigen einzulassen, dass uns paradoxerweise durch den Blick aus der Distanz, dem Anblick der Erde aus dem All, dieses kostbaren, verwundbaren Planeten, nahe gebracht worden ist?

Offen ist, inwieweit und wie unsere modernen neu auftretenden Seuchen, die „newly emerging infections“, wie auch die Rückkehr bekannter Infektionskrankheiten wie der Tuberkulose (,reemerging infections“), fruchtbar in einen solchen Kontext gestellt werden können und sollten. In wieweit sind beispielsweise EHEC/STEC-Infektionen eines neuen Typs rein mikrobiologische Phänomene? Wirft ein konsequentes Denken in Systemen und Ursachenketten, ein mehrmaliges „warum?“, nicht vielleicht auch hier unangenehme, dabei buchstäblich Not-wendige Fragen auf: Fragen nach der Vulnerabilität des uns tragenden Ökosystems, nach den Grenzen der Produktion tierischer Nahrungsmittel zur sicheren und ausreichenden Ernährung einer sich ausweitenden Menschheit, nach Nachhaltigkeit und Verteilungsgerechtigkeit in globaler Sicht? Nach Verantwortung gegenüber dem allgemeinen Gut genauso wie nach der Verantwortung gegenüber künftigen Generationen? Zur Verteilung von für die Gesundheit verfügbaren Mitteln zwischen den individualmedizinisch-kurativen und den bevölkerungsmedizi- nisch-präventiven Akteuren? Freiheit erfüllt und begründet sich nicht in Willkür, sondern in einem jeweils persönlich und gesellschaftlich ausbuchstabierten Ja zu Vernunft, Gerechtigkeit und Mitmenschlichkeit. Künftige Gesellschaften werden sich dieser Frage bewusst sein müssen, um überhaupt noch weiter Zukunft gestalten zu können - fast möchte man das Fazit derart auf den Punkt bringen.

Auch dieses Heft widmet sich mit seinen Themen wieder systemischen Fragen zu Gesundheit und Krankheit: dem Zusammenhang von Wohlfahrtspolitik und Gesundheit, den Chancen eines neuen Gewässermanagements, Pflegeheimen und Arbeitsunfähigkeit, ärztlichen Gutachten und Gewahrsamstauglichkeitsuntersuchungen - und nicht zuletzt dem Themenkomplex der Umgebungsuntersuchungen bei Tuberkulose einschließlich neuer diagnostischer Ansätze und der neuen Empfehlungen des Deutschen Zentralkommitees zur Bekämpfung der Tuberkulose.

Welche Gedanken Virchow zu diesen Themen gehabt hätte, bleibt Spekulation. 1849 verlor der revolutionär umtriebige Rudolf Virchow seine Anstellung in Berlin und fasst gleichzeitig seine Gedanken über „Die Seuche“ zusammen: „Die Entwickelung der Kultur [...] complicirt auch die Lebensbedingungen, und es ist gewöhnlich erst das Resultat langer und vielfach vergeblicher Bestrebungen, dass man endlich die complicirten Bedingungen so ordnet und ausgleicht, dass ihre Summen den natürlichen Lebensbedingungen äquivalent sind“ [6]. Vielleicht kann unser modernes Denken hier anknüpfen - wenn nicht als Revolution, so doch als Evolution im Gefolge eines Impulse gebenden, intelligenten, wissenschaftlich informierten gesellschaftlichen Diskurses („nudging“).

\section{Literatur}

1 RKI-Ratgeber für Ärzte. Typhus abdominalis, Paratyphus. Stand 9.1.2008. URL: http://www.rki.de/cln_178/nn_504582/DE/Content/ Infekt/EpidBull/Merkblaetter/Ratgeber_Mbl_Typhus_Paratyphus. html; Zugriff am 5.6.2011

2 Gottlieb MS, Schanker HM, Fan PT et al. Pneumocystis Pneumonia - Los Angeles. MMWR 1981; 30 (21): 1-3 URL: http://www.cdc.gov/mmwr/ preview/mmwrhtml/june_5.htm; Zugriff am 5.6.2011

3 Robert Koch-Institut. HIV-Infektionen und AIDS-Erkrankungen in Deutschland. Epidem Bull 2011; 21: 179-190

4 Gerabek WE. Ostdeutsche Biografie: Rudolf Virchow. URLhttp://www. ostdeutsche-biographie.de/vircru02.htm; Zugriff am 5.6.2011

5 Virchow R. Der Armenarzt. Aus: Die medicinische Reform. Nr. 18, 3.11.1848. S. 125

6 Virchow R. Die Einheitsbestrebungen in der wissenschaftlichen Medizin. (1849) In: Virchow $R$. Gesammelte Abhandlungen zur Wissenschaftlichen Medizin (2. unveränderte Ausgabe). Die Seuche. Hamm, Grotesche Buchhandlung; 1862; S. 55

7 JAMA. Health and human rights: a call to action on the $50^{\text {th }}$ anniversary of the Universal Declaration of Human Rights. The Writing Group for the Consortium for Health and Human Rights. JAMA 1998; 280 (05): 469-470 